\title{
Bitcoin: ¿Tenerla o ignorarla? Una aproximación hacia el tratamiento tributario peruano de la criptomoneda más famosa del mundo ${ }^{(*)}$
}

\section{Bitcoin: Have it or ignore it? An approach to Peruvian tax treatment of the most famous cryptocurrency in the world}

\author{
Lisset López Miranda ${ }^{(*)}$ \\ Payet, Rey, Cauvi, Pérez Abogados
}

"If you guys want proof Bitcoin is real, send them to me, I'll cash them out and feed homeless people." - Jason King

(Hacker humanitario) $^{(1)}$

\begin{abstract}
Resumen: El Perú no es un país ajeno al boom de las criptomonedas como mecanismos consensuados de pago e instrumentos de inversión. Sin embargo, en la actualidad no existe un marco normativo que desarrolle expresamente las consecuencias legales $\mathrm{ni}$ tributarias de las operaciones con Bitcoins $u$ otras criptomonedas. La autora desarrolla un análisis jurídico tributario aplicable en el Perú a las operaciones vinculadas a los Bitcoins: minado, intercambio por bienes y servicios, y transacciones de criptomonedas por divisas de curso legal. Así, partiendo de la definición de la naturaleza jurídica de las criptomonedas sobre la base de la legislación peruana vigente, y sin perder de vista a la experiencia del Derecho comparado, la autora perfila las implicancias fiscales del mundo de las criptomonedas en el Perú.
\end{abstract}

Palabras clave: Derecho Tributario - Bitcoins - Criptomonedas - Minado - Transferencia - Regulación legal - Valor mobiliario - Dinero electrónico - Intercambio de criptomonedas - Tratamiento tributario - Impuesto General a las Ventas - Impuesto a la Renta - Permuta - Bien mueble - Bien intangible - Perú

\begin{abstract}
The boom of cryptocurrencies as a mechanism of payment and investment has reached Peru, but our country lacks a legal regulation regarding the legal and fiscal consequences of operations with Bitcoins or other kinds of cryptocurrencies. In this article, the author elaborates a legal-fiscal analysis applicable to Bitcoin-related operations in Peru: mining, exchanges for goods and services, and exchanges for other kinds of currency. The first part of this article questions the legal nature of criptocurrencies based on the Peruvian legislation in force, while also taking into account the experiences of foreign legal systems. Thus, in the second part, the author describes the fiscal implications of criptocurencies in Peru.
\end{abstract}

Keywords: Tax Law - Bitcoins - Cryptocurrency - Mining - Transfer - Legal regulation - Securities - Digital money - Cryptocurrency exchanges - Tax treatment - Sales tax Income tax - Good exchange - Movable property - Intangible assets - Peru

$\left(^{*}\right) \quad$ Nota del Editor: el artículo fue recibido el 1 de marzo y aprobada su publicación el 7 mayo de 2019.

${ }^{* *}$ Abogada por la Pontificia Universidad Católica del Perú. Ha realizado LL.M. en Tributación International en el Levin College School of Law de la Universidad de Florida, Gainesville. Asociada senior del área tributaria del Estudio Payet, Rey, Cauvi, Pérez Abogados. Actualmente es profesora de cursos de tributación en la Facultad de Derecho de la Universidad de Lima (pregrado), y en la Facultad de Derecho de la Universidad San Martín de Porres (postgrado). Correo electrónico: Ilm@prcp.com.pe

(1) Hacker humanitario, co-fundador de Kingsland University, School of Blockchain. 
Bitcoin: ¿Tenerla o ignorarla? Una aproximación hacia el tratamiento tributario peruano de la criptomoneda más famosa del mundo

Bitcoin: Have it or ignore it? An approach to Peruvian tax treatment of the most famous cryptocurrency in the world

\section{Introducción}

A marzo de 2019, existen más de cincuenta establecimientos en el Perú, treinta en Lima y los demás en otras nueve regiones, que aceptan a los Bitcoins como medio de pago de sus productos o servicios (restaurantes, hospedajes, servicios de turismo, vestido, dentistas, entre otros) ${ }^{(2)}$.

Cabe indicar que dicho número de establecimientos se ha incrementado en casi un $50 \%$ respecto a enero de $2018^{(3)}$. $Y$ es que nuestro país no es ajeno a la tendencia mundial del uso de las coloquialmente llamadas monedas virtuales o criptomonedas como innovadores, libres de costos de transacción excesivos y seguros mecanismos de pago para un sinfín de bienes y servicios.

De igual forma, el incentivo en la inversión en criptomonedas en general, y en el Bitcoin en particular, es su valor de cotización a nivel mundial. Así, el uso de las criptomonedas no solo se restringe a un medio de pago consensuado entre partes, sino que su potencial empleo como instrumento de especulación (o generador de ganancias de capital) viene ocupando la atención preponderante en el mercado actual.

En el a veces enigmático, pero fascinante mundo de las criptomonedas a ojos del jurista, la más famosa y cuyo valor de cotización bursátil al cierre del año 2017 supuso un incremento de su valor de 2,472\% respecto al cierre de 2016 (llegando casi a un valor de USD 20,000 por unidad de Bitcoin) $)^{(4)}$, es indudablemente, el Bitcoin.

Como quiera que por definición la realidad sobrepasa al Derecho, la regulación legal en general, y la tributaria, en especial, aplicable a los Bitcoin a nivel internacional dista mucho de ser uniforme. En el Perú, peor aún, nos encontramos ante la ausencia absoluta de regulación legal especial para este fenómeno tecnológico.

Bajo este escenario, el propósito de este artículo es marcar un punto de inicio para el análisis del tratamiento tributario en el Perú aplicable a las operaciones con presencia de Bitcoins o cualquier otra criptomoneda.
En consecuencia, como tratándose de cualquier escrutinio legal, el tratamiento tributario de las operaciones con Bitcoins dependerá esencialmente de la calificación o definición de la naturaleza jurídica de estos en el Perú. Para estos efectos, haremos uso de la legislación nacional, sin perder de vista a la experiencia de la legislación comparada. Solo tras ello, podremos aterrizar en el análisis tributario.

Finalmente, toda vez que el tratamiento legal y tributario en territorio peruano no debería variar materialmente dependiendo de una criptomoneda en particular, nuestro análisis sobre el Bitcoin resulta igualmente aplicable para las operaciones semejantes que se realicen con las demás criptomonedas que existen en el mercado (Ether, EOS, Ripple, Litecoin, Dogecoin, entre otras).

\section{Elementos básicos de los Bitcoin y de las criptomonedas en general}

\section{1 ¿Qué son los Bitcoins?}

De acuerdo con el Grupo de Acción Financiera (en adelante, "GAFI"), una moneda virtual es una representación digital de valor que puede ser comercializada digitalmente, y, que, debido al consenso de la comunidad de sus usuarios, funciona como lo siguiente: (i) un medio de cambio, (ii) una unidad de cuenta, y (iii) un depósito de valor. Sin embargo, ninguna jurisdicción emite o garantiza a las monedas virtuales ${ }^{(5)}$.

Con respecto al Bitcoin, esta es la primera moneda virtual considerada como criptomoneda ${ }^{(6)}$,basada en "(...) un programa

(2) Coinmap.org, Map of Bitcoin accepting venues, Sitio web de Coinmap.org,https://coinmap.org/\#/map/-11.94125750/-76.57470703/10 (consultada el 1 de marzo de 2019). A inicios de 2018, el número de estos establecimientos en el Perú ascendía a veintisiete.

(3) Véase Lucero Chávez, "Bitcoin: Estos negocios peruanos aceptan pagos con ellos", sitio web de El Comercio, https://elcomercio. pe/economia/mercados/bitcoin-negocios-peruanos-puedes-pagar-noticia-488677.

(4) Alberto Gil Soriano, "Monedas virtuales: Aproximación jurídico - tributaria y control tributario”,Actualidad Jurídica Uría Menéndez, https://www.uria.com/documentos/publicaciones/5803/documento/foro_esp_02.pdf?id=7879 (consultada el 10 de enero de 2019). Al 4 de marzo de 2019, el valor promedio de cotización por Bitcoin (BTC) según el Exchange Local Bitcoin es de USD 4000 dólares o S/12400 soles. Sitio web de LocalBitcoins.com, https://localbitcoins.com/?ch=4796 (consultada el 4 de marzo de 2019).

(5) Grupo de Acción Financiera (GAFI),"Informe del GAFI. Monedas Virtuales: Definiciones claves y riesgos potenciales de LA/FT", [https://www.uaf.cl/asuntos/descargar.aspx?arid=961].

(6) Se entiende como criptomoneda a una moneda virtual fundamentada matemáticamente y que está protegida por criptografía (ciencia de escribir mensajes en forma cifrada o en código)," (...) para implementar una economía de la información segura, descentralizada y distribuida. Las criptomonedas se basan en llaves públicas y privadas para transferir el valor de una persona (individuo o entidad) a otra, y debe ser criptográficamente firmado cada vez que se transfiere". Definición brindada por el GAFI, en GAFI, "Informe del GAFI. Monedas Virtuales: Definiciones claves y riesgos potenciales de LA/FT". 
(software) libre. Dicho programa pone a disposición de sus usuarios su código fuente con el fin de que estos puedan usarlo, copiarlo, modificarlo y redistribuirlo de modo que se pueden crear nuevos proyectos de software libre a partir de él" (Gutiérrez y Moreno 2018, 27).

De esta forma, "el sistema Bitcoin se rige, de un lado, por medio de un protocolo informático, encargado de establecer las directrices para la creación y transferencia de Bitcoins y, de otro, por una red peer-to-peer o usuario a usuario, conocida en inglés como 'blockchain', que permite 'el intercambio directo de información, en cualquier formato, entre todos los ordenadores interconectados' en una red muy numerosa y compleja" (Ramírez 2018, 8).

Vale añadir que el sistema Bitcoin nació con un número de emisión máxima de unidades de estas criptomonedas. Así, desde su creación (año 2008) el sistema está diseñado para emitir solo 21 millones de Bitcoins, cuya fecha límite es el 7 de mayo de 2140. A marzo de 2019, se encuentran en circulación 17.5 millones de Bitcoins ( $81 \%$ de la meta) que representan un mercado aproximado de USD 66,062 millones $^{(7)}$.

Asimismo, cabe enfatizar que los Bitcoins y las criptomonedas en general, funcionan económicamente como medio de pago pues a través de ellos se pueden adquirir bienes y servicios en los establecimientos y/o proveedores que han aceptado recibir pagos en dicha moneda virtual. Asimismo, su valor es altamente volátil, dado que no se encuentran respaldados por ningún Estado o entidad privada, y que han sido usados de forma especulativa por muchos inversionistas en los años recientes.

\section{2 ¿Cómo se obtiene un Bitcoin?}

Al respecto, son tres las principales formas de obtener un Bitcoin, a saber:

a) Aceptando Bitcoins como medio de pago; según adelantamos, en la actualidad muchos establecimientos comerciales, sean físicos o digitales, aceptan esta moneda como medio de pago para transar bienes.

En el Perú son más de cincuenta los establecimientos que aceptan Bitcoins como medios de pago y servicios. Asimismo, tenemos conocimiento que con mayor frecuencia diversas sociedades peruanas y extranjeras (sobre todo las denominadas empresas Fintech ${ }^{(8)}$ ) compensan a sus colaboradores, personal dependiente e independiente, mediante Bitcoins (o fracciones de estas, como son los Satoshi(9) $\mathrm{u}$ otras criptomonedas.

b) Produciendo Bitcoins; a este proceso se le conoce como minado de Bitcoins, y consiste en destinar gran potencia de procesamiento de un ordenador para resolver problemas matemáticos y realizar verificaciones de las transacciones de la criptomoneda. A cambio de ello, el propio sistema informático del Bitcoin emite estas a favor del minero ${ }^{(10)}$.

Es relevante agregar que el minado de Bitcoins requiere una importante inversión en equipos y sobre todo electricidad para soportar el trabajo de las computadoras de los mineros, que son las encargadas de resolver los complejos algoritmos matemáticos que el sistema Bitcoin propone.

A fin de conocer un poco más sobre el minado y de su retribución, debemos indicar que el sistema Bitcoin está diseñado para que, con el transcurso del tiempo, menos Bitcoins sean emitidos como recompensa por el minado, mientras que la complejidad de la verificación y validación de las transacciones sea cada vez mayor ${ }^{(11)}$. De esta forma, cada cuatro años el valor de recompensa se reduce a la mitad, siendo que actualmente la retribución es de 12.5 Bitcoin, y se ajustará a 6.25 Bitcoins en junio de 2020.

c) Adquiriendo Bitcoins en plataformas de intercambio o casas de cambio (Exchange

(7) Revisar: Redacción Gestión, "Solo queda el 20\% de Bitcoins por minar en el mundo", sitio web de Gestión, https://gestion.pe/ economia/queda-20-bitcoins-minar-mundo-225022.

(8) Las empresas conocidas como Fintech son aquellas que hacen de la tecnología su principal activo para prestar servicios financieros, tales como, medios de pago y transferencia, financiamiento, cambio de divisas, criptomonedas, finanzas personas y asesoría financiera, etc. A fin de tener una idea del estado de las Fintech en el Perú y la tendencia hacia la regulación de algunas de estas, véase el siguiente artículo de Gestión: Omar Manrique, "Se exigiría un capital mínimo a las fintech para que puedan operar", sitio web de Gestión,https://gestion.pe/economia/exigiria-capital-minimo-fintech-puedan-operar-257377?href=tepuedeinteresar.

(9) Un Satoshi es la mínima unidad en que la que puede dividir un Bitcoin ("BTC"), y se expresa como 0.00000001 Bitcoin.

(10) Según el GAFI, "un minero es una persona o entidad que participa en una red de moneda virtual descentralizada mediante la ejecución de un software especial para resolver complejos algoritmos en una prueba de trabajo distribuida u otro sistema de prueba distribuido utilizado para validad las transacciones en el sistema de moneda virtual”. En Grupo de Acción Financiera (GAFI), "Informe del GAFI. Monedas Virtuales: Definiciones claves y riesgos potenciales de LA/FT".

(11) Marcin Szczepanski, "Bitcoin: market, economics and regulations", European Parliamentary Research Service, http://www.europarl. europa.eu/RegData/bibliotheque/briefing/2014/140793/LDM_BRI2014)140793_REV1_EN.pdf (consultada el 20 de febrero de 2019). 
Bitcoin: ¿Tenerla o ignorarla? Una aproximación hacia el tratamiento tributario peruano de la criptomoneda más famosa del mundo

Bitcoin: Have it or ignore it? An approach to Peruvian tax treatment of the most famous cryptocurrency in the world

de criptomonedas); las monedas virtuales pueden ser adquiridas e intercambiadas en numerosas Exchange, en algunas de las cuales se transan operaciones entre más de una clase de criptomoneda, y más de una divisa de curso legal. La mayoría se trata de plataformas virtuales extranjeras, aunque la aparición de plataformas locales ${ }^{(12)}$ está en aumento.

Pueden acceder a los Exchange personas naturales 0 jurídicas, sea que inviertan en criptomonedas como reservas a largo plazo, o con fines especulativos aprovechando la baja del precio para su compra; y el alza, para su venta ${ }^{(13)}$.

\section{Naturaleza jurídica del Bitcoin}

La naturaleza jurídica del Bitcoin u otras monedas virtuales no ha sido definida por la legislación peruana y tampoco esta ha ensayado alguna propuesta de definición. No obstante, a continuación se analiza si es posible incluir a los Bitcoins (u otras criptomonedas) bajo algún concepto jurídico previsto normativamente en el Perú.

3.1 ¿Las criptomonedas califican como una moneda legal? Los Bitcoins no califican como moneda dado que no han sido emitidos por una autoridad gubernamental o banco central de un país determinado. Así, según fue explicado en el numeral 2.2 precedente, los Bitcoins son emitidos a través del proceso de minado realizado por personas o grupos de personas especializadas en descifrar los códigos requeridos para la validación de las transacciones realizadas en el sistema Bitcoin.

Por su parte, la emisión de billetes y monedas, dentro del territorio peruano, es facultad exclusiva del Estado Peruano, de acuerdo con el artículo 83 de la Constitución Política de 1993. Esta facultad es ejercida por intermedio del Banco Central de Reserva del Perú (en adelante, "BCRP").

\section{2 ¿Las criptomonedas pueden ser clasificadas como dinero electrónico?}

Los Bitcoins tampoco califican como dinero electrónico, dado que no cumplen con las características previstas en el artículo 2 de la ley que regula las características básicas del dinero electrónico como instrumento de inclusión financiera, aprobada por la Ley 29985.

De acuerdo con el referido artículo, el dinero electrónico es un valor monetario representado por un crédito exigible a su emisor, el cual tiene las siguientes características:

a) Es almacenado en un soporte electrónico. b) Es aceptado como medio de pago por entidades o personas distintas del emisor $y$ tiene efecto cancelatorio.

c) Es emitido por un valor igual a los fondos recibidos.

d) Es convertible a dinero en efectivo según el valor monetario del que disponga el titular, al valor nominal.

e) No constituye depósito y no genera intereses.

Así, los Bitcoins en particular (y las criptomonedas en general) no representan créditos exigibles a ningún emisor. Ello teniendo en cuenta que no son emitidos por una entidad sino por el propio sistema informático Bitcoin, en virtud del proceso de minado.

Igualmente, los Bitcoins no cumplen con las características indicadas en los literales c) y d) anteriores debido a que no se emiten como contraprestación por la entrega de fondos, ni tampoco son convertibles a dinero en efectivo a un valor nominal (no tienen un valor determinado, siendo que este fluctúa en función de la oferta y demanda existente por esta moneda virtual).

Coincidiendo con esta conclusión, el GAFI señala que, en efecto, "Ia moneda virtual es diferente del dinero electrónico puesto que éste es una representación digital del dinero fiduciario (de curso legal) usado electrónicamente para transferir el valor denominado en dinero fiduciario". Agrega que "el dinero electrónico funciona como un mecanismo de transferencia digital para el dinero fiduciario, es decir, se transfiere electrónicamente un valor que tiene la condición de moneda de curso legal" (GAFI 2014, 4).

\section{3 ¿Las criptomonedas califican como instrumento o medio de pago?}

De acuerdo con la Ley de los Sistemas de Pagos y de Liquidación de Valores, aprobada por la Ley 29440, los instrumentos de pago tienen por objeto efectuar un pago (como las transferencias de créditos) o requerirlo

(12) Dentro de las plataformas peruanas tenemos a Buda, Bitinka, SurBTC, LocalBitcoins, e inclusive existe un cajero automático de Bitcoins asociado a la plataforma Cajero.pe. Fuente: Andrea Leal, “¿Dónde comprar y vender bitcoins en Perú?”, sitio web de Criptonoticias, https://www.criptonoticias.com/colecciones/donde-comprar-vender-bitcoins-peru/.

(13) Entre los Exchange de criptomonedas más populares tenemos a Coinbase, LocalBitcoins, Changelly, Bittrex, CEXio, HitBTC, BitFinex. Fuente: CoinList, "Los Mejores Exchanges de Criptomonedas en 2019", sitio web de CoinList, https://coinlist.me/es/exchanges/. 
(como los cheques, débitos directos, letras de cambio, cuotas de crédito).

Ahora bien, los instrumentos de pago indicados en la Ley 29440 son aquellos utilizados en el marco de un sistema de pagos o de liquidación de valores, los cuales se encuentran reconocidos de manera expresa por la referida ley o son declarados como tales por el BCRP o la Superintendencia del Mercado de Valores (en adelante, "SMV"), respectivamente.

Teniendo en cuenta que el sistema Bitcoin a la fecha no ha sido reconocido por la legislación peruana o declarado por el BCRP, órgano rector de los sistemas de pagos, como un sistema de pagos, los Bitcoins tampoco constituyen instrumentos de pago bajo la Ley 29440.

Sin perjuicio de lo mencionado, los Bitcoins pueden ser utilizados como medios o mecanismos de pago de forma consuetudinaria, en efecto, en los establecimientos que las aceptan, estas criptomonedas gozan de efecto cancelatorio respecto de los bienes y servicios prestados. Dicho efecto cancelatorio es concedido de manera voluntaria por las partes involucradas en cada transacción, lo cual se asemejaría a una dación en pago.

\section{4 ¿Las criptomonedas son commodities?}

De acuerdo con el Glosario de la Ley General del Sistema Financiero y del Sistema de Seguros y Orgánica de la Superintendencia de Banca, Seguros y AFP, aprobada por la Ley 26702, los commodities son mercancías primarias o básicas consistentes en productos físicos, que pueden ser intercambiados en un mercado secundario, incluyendo metales preciosos, pero excluyendo al oro, que es tratado como una divisa.

En tal sentido, teniendo en cuenta que las monedas virtuales solo se encuentran representadas digitalmente, y no de manera física, no califican como commodities.

\section{5 ¿Las criptomonedas pueden ser considerados como títulos valores o valores mobiliarios?}

De acuerdo con el artículo 1 de la Ley de Títulos Valores, aprobada por la Ley 27287 , los valores materializados que representen o incorporen derechos patrimoniales, tendrán la calidad y los efectos de título valor, cuando estén destinados a la circulación, siempre que reúnan los requisitos formales esenciales que, por imperio de la ley, les corresponda según su naturaleza.

Así, se denomina títulos valores a un conjunto de documentos típicos (o predeterminados), como letras de cambio, pagarés, cheques, certificados de depósitos, vales de prenda, acciones de sociedades, obligaciones, cartas de porte, conocimientos de embarque, etc., que contribuyen a promover la actividad económica, agilizando y dando fluidez al tráfico patrimonial (Montoya 2004, 3).

Teniendo en cuenta que los títulos valores son documentos típicos que deben haber sido reconocidos como tales expresamente por ley, y que ello no ha ocurrido con respecto a ninguna moneda virtual, estas no califican como títulos valores.

Por la misma razón, las monedas virtuales tampoco califican como valores mobiliarios, considerando que estos son una subespecie de títulos valores, de acuerdo con lo previsto en el artículo 255 de la Ley de Títulos Valores.

Además, debe tenerse en cuenta que, de conformidad con el artículo 3 del Texto Único Ordenado de la Ley del Mercado de Valores, aprobado por Decreto Supremo 093-2002$\mathrm{EF}$, son valores mobiliarios aquellos emitidos en forma masiva y libremente negociables que confieren a sus titulares derechos crediticios, dominiales o patrimoniales, o los de participación en el capital, el patrimonio o las utilidades del emisor.

Por tanto, teniendo en cuenta que los Bitcoins no son emitidos por entidad centralizada, sino por el propio sistema informático Bitcoin, una vez culminado el respectivo proceso de minado, tampoco calificarían como valores mobiliarios en función de la definición de estos antes indicada. La misma conclusión aplica para toda clase de criptomoneda.

\section{6 ¿Las criptomonedas califican como instrumento $o$ activo financiero?}

Si bien no existen definiciones bajo la normativa de la Superintendencia de Banca, Seguros y AFP (en adelante, "SBS") o de la SMV sobre lo que debe entenderse por instrumento financiero o activo financiero ${ }^{(14)}$, ambos conceptos han sido definidos por la

(14) Sin perjuicio de ello, de acuerdo con el artículo 2 de la Ley de Promoción del Mercado de Valores, aprobada por la Ley 30050 , toda publicidad $u$ ofrecimiento de compra o venta o suscripción de activos financieros que se realice en territorio nacional empleando medios masivos de comunicación, como diarios, revistas, radio, televisión, correo, reuniones, redes sociales, servidores de Internet ubicados en territorio nacional u otros medios o plataformas tecnológicas, solo puede realizarse por sujetos autorizados o supervisados por la SMV o por la SBS. 
Bitcoin: ¿Tenerla o ignorarla? Una aproximación hacia el tratamiento tributario peruano de la criptomoneda más famosa del mundo

Bitcoin: Have it or ignore it? An approach to Peruvian tax treatment of the most famous cryptocurrency in the world

Norma Internacional de Contabilidad (en adelante, "NIC") 32, adoptada por el Ministerio de Economía y Finanzas.

Así, de acuerdo con la NIC 32, un instrumento financiero es cualquier contrato que dé lugar a un activo financiero en una entidad y a un pasivo financiero o a un instrumento de patrimonio en otra entidad.

Por su parte, un activo financiero es cualquier activo que sea: (a) efectivo; (b) un instrumento de patrimonio de otra entidad; (c) un derecho contractual: (i) a recibir efectivo $\mathrm{u}$ otro activo financiero de otra entidad; o (ii) a intercambiar activos financieros o pasivos financieros con otra entidad, en condiciones que sean potencialmente favorables para la entidad; o (d) un contrato que será o podrá ser liquidado utilizando instrumentos de patrimonio propio de la entidad, y sea: (i) un instrumento no derivado, según el cual la entidad está o puede estar obligada a recibir una cantidad variable de sus instrumentos de patrimonio propios; o (ii) un instrumento derivado que será o podrá ser liquidado mediante una forma distinta al intercambio de un importe fijo de efectivo, o de otro activo financiero, por una cantidad fija de los instrumentos de patrimonio propio de la entidad.

Como puede apreciarse, teniendo en cuenta que los Bitcoins no constituyen efectivo, ni un contrato, ni dan lugar a un instrumento de patrimonio o a un derecho contractual, no constituyen instrumentos o activos financieros bajo la NIC 32 .

3.7 ¿Las criptomonedas califican como bienes muebles? Teniendo en cuenta que no es posible calificar a los Bitcoins ni a ninguna otra criptomoneda bajo ninguno de los conceptos jurídicos anteriormente indicados, consideramos que estos deben ser clasificados bajo la categoría general de bienes muebles previstos en el artículo 886 del Código Civil.

En efecto, según el numeral 10 del artículo 886 del Código Civil Peruano, son considerados bienes muebles todos aquellos bienes no comprendidos en el artículo que corresponde al listado de bienes inmuebles (artículo 885). De tal forma que, al igual que los derechos de autor, marcas y patentes, las criptomonedas son bienes muebles para efectos de la legislación peruana.

Así, tomando en cuenta el ordenamiento legal peruano vigente, que no cuenta con una regulación específica para el Bitcoin, ni para las demás criptomonedas, podemos afirmar que el Bitcoin es un bien incorporal calificado como bien mueble según el Código Civil (Gutiérrez y Moreno 2018, 50).

\section{8 ¿Cómo se regulan o clasifican las monedas virtuales en otros países?}

La regulación de las criptomonedas es diversa a nivel internacional. Algunos países como Bolivia, Rusia, India,
Tailandia, Ecuador, entre otros, han prohibido o restringido severamente el uso de criptomonedas, negando por ejemplo su uso como medio de pago (Ramírez 2018, 22).

De otro lado, países como Alemania reconocen al Bitcoin como un instrumento financiero que, si bien carece del estatus de una moneda de curso legal, funciona como una unidad de cuenta y tiene similitudes al dinero privado o regional. Similar al caso alemán, el Banco Central de Inglaterra ha afirmado que los Bitcoins actuarían también como dinero privado o moneda extranjera (Ramírez 2018, 14-15).

De otro lado, muchos países, como Francia y Alemania, han aclarado que los proveedores de monedas virtuales se encuentran comprendidos en los requerimientos existentes para las licencias de proveedores de servicios de pagos y financieros (Sotiropoulou y Guégan 2017, 475).

Tratándose de Estados Unidos, Alberto Gil menciona que, "para la US Securities Exchange Commision (SEC), las monedas virtuales son un contrato de inversión (investment contract), y como tal, tienen la calidad de instrumento financiero (o security)" (Gil 2018, 74).

En el caso de Brasil, a partir de 2014, las criptomonedas son incluidas en la categoría de activos financieros. Por su parte, para Canadá, los Bitcoins no son calificados como moneda de curso legal, sino como mercancía (Ramírez 2018, 21).

Como hemos indicado, en la actualidad la legislación peruana no ha establecido ningún tipo de regulación legal(15) (financiera, tributaria, penal, etc.) con relación a las criptomonedas, a los mercados secundarios en los cuales estas son transadas, ni a los intermediarios y demás participantes de dichos mercados.

No obstante, no podemos descartar que se avizore pronto una regulación legal de las criptomonedas, ello en la medida que estas adquieran mayor importancia en nuestro país, dado el volumen de operaciones con

(15) Cabe indicar que desconocemos de la existencia de algún proyecto de ley u otra iniciativa para regular a las transacciones y en general, todo lo vinculado a las criptomonedas. 
monedas virtuales, el uso masivo de Exchanges locales o no de criptomonedas, y el incremento del número de establecimientos que las acepten como medio de pago.

En específico, existe un importante interés a nivel internacional por impulsar el control regulatorio de las operaciones con criptomonedas a fin de evitar el lavado de activos y financiamiento del terrorismo, considerando que uno de los atractivos principales de estas monedas virtuales es el anonimato y la seguridad para sus usuarios (Szczepanski 2014, 4).

\section{Tratamiento tributario de las criptomonedas en el Perú}

En primer lugar, debemos señalar que la legislación tributaria peruana no ha regulado aún de manera expresa las implicancias tributarias vinculadas al ciclo de las criptomonedas: producción, intercambio, y venta. De este modo, y según indicamos en la introducción del presente artículo, lo esencial para determinar las consecuencias fiscales de las operaciones vinculadas a las criptomonedas bajo las normas vigentes, dependerá de la naturaleza jurídica de tales bienes.

Así, de acuerdo con la sección 3.7 del presente artículo, hemos llegado a la conclusión que los Bitcoins son bienes incorporales que gozan de la calidad de bienes muebles. Bajo este marco, analizaremos a continuación las consecuencias fiscales de la producción, intercambio y venta de Bitcoins (o criptomonedas en general) en el Perú.

\subsection{Implicancias tributarias de la producción o minado de criptomonedas desde Perú}

Conforme lo indicado en la sección 2.2., el minado de criptomonedas supone la resolución de algoritmos matemáticos propuestos por el sistema Bitcoin, dicha resolución supone un requisito para la validación de las transacciones con Bitcoins, lo cual garantiza su continuidad.

Una vez realizada la labor del minero, este recibirá en compensación unidades de Bitcoins emitidos por el mismo sistema.

\subsubsection{Impuesto a la Renta}

Sobre el particular, considerando a una sociedad domiciliada que ejerce la función de minero de Bitcoins (o de criptomonedas que tengan una forma similar de generación) mediante un data center ubicado en el Perú, esta actividad sería calificada como empresarial pues implica la explotación de un negocio; y en consecuencia, gravada con el Impuesto a la Renta (en adelante, "IR") en el Perú, involucrando los factores capital más trabajo.

De otro lado, veamos el caso de una persona natural domiciliada, supongamos que una persona experta en informática, que mediante la adquisición de potentes servidores ubicados en el Perú se dedica de forma individual al minado de Bitcoins, y recibe por sus servicios al sistema unidades de esta moneda.

Al respecto, somos de la opinión que dicha el pago en Bitcoins constituye la compensación por el ejercicio individual de su profesión o ciencia, mas no una actividad empresarial(16). En consecuencia, se trataría de un servicio que genera rentas de cuarta categoría.

Ahora bien, la particularidad del gravamen a las actividades de minado de Bitcoins es que el ingreso gravado tanto para una sociedad como para el minero persona natural independiente, sería la suma del valor de mercado de cada Bitcoin que se obtenga por el proceso de minado.

En efecto, ello toda vez que la asignación de unidades de Bitcoins por el sistema es la contraprestación que se recibe por el desarrollo de algoritmos matemáticos que facilitarán la cadena de intercambio (o blockchain) que representan los Bitcoins. Dicho tratamiento tributario es el aplicado por países como Estados Unidos y España(17).

Sobre el particular, no existen reglas que definan el valor de mercado de bienes como los Bitcoins, y más bien las normas reglamentarias del IR en el caso del valor de mercado de valores (entendemos que hace referencia a los valores mobiliarios)

(16) Consideramos que el contar con algunos elementos de capital para el desarrollo del minado no conlleva a que la persona natural que realiza individualmente dicha actividad sea calificada como un empresario; y por ende, generador de renta empresarial. Ciertamente, opinamos que es razonable que, para las actividades de profesionales independientes, los individuos sumen a su trabajo la inversión en capital para el desempeño de sus servicios (materiales e instrumentos) sin devenir en empresarios.

(17) Véase: Rogelio Villalba García, "Impuestos y tributación de bitcoin y criptomonedas", sitio web de Asepyme, https://asepyme.com/ impuestos-y-tributacion-de-bitcoin-y-criptomonedas-irpf-iva-itp-ip-is/\#Obligaciones_y_tributacion_de_los_mineros_de_bitcoin_o_ criptomonedaspara España, y http://theminersunion.com/2017/09/11/what-you-need-to-know-about-bitcoin-mining-taxes/ para Estados Unidos. 
Bitcoin: ¿Tenerla o ignorarla? Una aproximación hacia el tratamiento tributario peruano de la criptomoneda más famosa del mundo

Bitcoin: Have it or ignore it? An approach to Peruvian tax treatment of the most famous cryptocurrency in the world

que cotizan varias bolsas o mecanismos centralizados de negociación, señalan que el valor de mercado a considerar será el mayor valor de cotización.

Así también y de manera referencial, tanto en el caso de existencias como de activos fijos, el criterio del valor de mercado preponderante es el que se refiere al valor que se obtiene en operaciones onerosas con terceros o en transacciones frecuentes, lo que nos puede llevar también a considerar como valor de mercado de los Bitcoins a los valores de cotización de estos en el mercado mundial (Exchange de criptomonedas).

De esta forma, ante la ausencia de norma expresa, quedaría la incertidumbre sobre qué valor de cotización se debe emplear en un contexto en el cual existen varios miles de Exchange a nivel mundial, los que pueden presentar diferencias de valor de los Bitcoins en una misma fecha (la de asignación de Bitcoins al minero).

Paralelamente, debemos indicar que el valor de cotización del Bitcoin que se utilice representará a su vez el costo computable de los mismos Bitcoins para una futura enajenación de estos por parte del minero (persona natural o jurídica).

De otro lado, en el caso de sociedades dedicadas al minado, los gastos deducibles para la determinación de la renta neta serán entre otros, la energía eléctrica empleada para el minado, la depreciación de los equipos informáticos empleados, planillas, arrendamiento de locales especiales, etc.

En el caso de mineros personas naturales estos no podrán deducir dichos gastos reales, pues la deducción reconocida para estos casos de servicios que constituyan rentas de cuarta categoría $^{(18)}$, causando que dicha actividad pueda no resultarles rentable desde un punto de vista fiscal.

\subsubsection{Impuesto General a las Ventas}

Conforme al artículo 1 de la Ley del Impuesto General a las Ventas (en adelante, "IGV"), se encuentra gravado con dicho impuesto, entre otros, la prestación de servicios en el país.

Complementando la norma anterior, el literal c) del artículo 3 de la Ley del IGV establece que se considera servicio a "toda prestación que una persona realiza para otra y por la cual percibe una retribución o ingreso que se considere renta de tercera categoría para los efectos del Impuesto a la Renta, aun cuando no esté afecto a este último impuesto; incluidos el arrendamiento de bienes muebles e inmuebles y el arrendamiento financiero".

Respecto al contribuyente del IGV, el artículo 9 de la Ley del IGV señala que tienen tal calidad aquellas personas naturales o jurídicas que desarrollan actividad empresarial que se encuentre en el ámbito de las operaciones gravadas con este impuesto.

De esta forma, una persona natural domiciliada que realice el minado de Bitcoins no realizaría actividad empresarial; y, por tanto, estará fuera del ámbito de aplicación del IGV. En tal sentido, nos referiremos en este acápite solo al caso de una persona jurídica que se dedica al minado de Bitcoins.

Aplicando la definición de servicio a nuestro caso concreto, la materia de discusión para el minado de criptomonedas es si dicha actividad implica que una sociedad domiciliada cumpla con la parte de la norma que prescribe que el servicio es una "prestación que una persona realiza para otra". Por tanto, corresponder determinar si el minado consiste una prestación, y si esta se da a un tercero (exigencia de alteridad).

En efecto, lo primero a evaluar es si el minado determina una prestación. Sobre esto, la definición tradicional de prestación es un comportamiento o conducta que consiste en un dar (que no implique transferencia de propiedad), un hacer o un no hacer. De ello podemos inferir que la definición de "prestación" sería lo suficientemente amplia como para abarcar a la realización de la actividad de resolver algoritmos matemáticos en el sistema de Bitcoins.

De otro lado, respecto al requisito de alteridad o de pluralidad de partes en la prestación gravada con el IGV, el tema que se presenta en el minado de criptomonedas es que no es posible identificar a un sujeto concreto (entidad o persona natural) beneficiario de la prestación definida (resolución de problemas matemáticos del blockchain), ni al pagador de la retribución, toda vez que esta (emisión de Bitcoins) es generada automáticamente por un sistema informático.

Sobre lo anterior, entendemos que la Administración Tributaria peruana (a nivel de SUNAT y del Tribunal Fiscal) no se ha

(18) Ello dado que para la determinación de la renta de cuarta categoría solo se aplica una deducción fija del $20 \%$ de la renta bruta. Asimismo, para el cálculo de la renta neta del trabajo se deducen 7 UIT, y otras 3 UIT para determinados casos, deducciones que no cubrirían los altos costos de la actividad del minado para una persona natural. 
pronunciado en un caso semejante. Asimismo, se podrían presentar dificultades prácticas que el gravamen con el IGV al minado de Bitcoins generaría como la emisión del comprobante de pago por el servicio (¿a nombre de quién se consignaría el comprobante?).

Cabe indicar que en países con legislaciones semejantes a la peruana en cuanto al IGV (o IVA en Europa), como España, la autoridad fiscal ha establecido de forma expresa que la actividad que realiza el minero de Bitcoins no está sujeta al IVA, ya que el servicio que presta no lo hace a un usuario determinado, pues no tiene ninguna relación con ningún usuario de la blockchain. Así lo ha indicado expresamente la Resolución Vinculante de la Dirección General de Tributos, V3625-16 del 31 de agosto de $2016^{(19)}$.

Bajo este escenario un poco incierto, no podemos descartar que se exija al minero persona jurídica que grave con el IGV del $18 \%$ sobre la contraprestación por el minado, esto es, el valor de mercado de las Bitcoins emitidas en cada mes ${ }^{(20)}$.

\subsection{Implicancias tributarias de la transferencia de criptomonedas en calidad de medio de pago}

Según lo indicado anteriormente, bajo el ordenamiento legal peruano las criptomonedas calificarían como bienes muebles (distintos a valores mobiliarios, dinero, título valor u otros). De esta forma, cualquier intercambio de bienes por criptomonedas en calidad de medio de pago generará una permuta para efectos legales.

\subsubsection{Impuesto a la Renta (IR)}

La permuta constituye un supuesto de enajenación de bienes gravado con el IR, sujeta a las reglas de valor de mercado. Así tenemos que, si una sociedad domiciliada adquiere bienes mediante la transferencia de Bitcoins que disponga en su poder (en su monedero digital o wallet), dicha operación supone reconocer dos transferencias que podrían estar gravadas con el IR:

- La transferencia del Bitcoin generará renta gravada para su transferente (la sociedad poseedora de la criptomoneda) sobre la diferencia entre el valor de transferencia (el valor de mercado del bien recibido), menos el costo computable del Bitcoin (el que dependerá de si para su obtención se hizo uso de minado, de un intercambio o compra en Exchange). La tasa del IR aplicable a la sociedad será del $29.5 \%$ sobre renta neta anual.

- La transferencia del bien con el cual el Bitcoin se intercambia producirá una renta gravada en el Perú solo si se trata de renta gravada para su transferente, la cual se determinará por la diferencia entre el valor de mercado del Bitcoin recibido y el costo computable del bien transferido.

Asimismo, en aplicación del numeral $7 \mathrm{del}$ artículo 21 de la Ley del IR, el costo computable de los bienes recibidos en una permuta será el valor de mercado de dichos bienes.

Mención aparte merece el caso de Bitcoins que son transferidas por personas naturales domiciliadas (que no realizan actividad empresarial vinculada a las criptomonedas) e intercambiados por bienes o servicios. Dicha operación no estará gravada con el IR en Perú, dado estas personas solo generan ganancias de capital gravadas en el caso de venta de inmuebles y de valores mobiliarios (calificación que no gozan los Bitcoins, según explicamos en el numeral 3.5 precedente).

4.2.2 Impuesto General a las Ventas (IGV) De acuerdo con la Ley del IGV, la venta en el país de bienes muebles se encuentra gravado con dicho impuesto. Para estos efectos, toda transferencia de propiedad de bienes calificará como una venta.

Ahora bien, el literal b) del artículo 3 de la Ley del IGV reconoce como bienes muebles a aquellos corporales que pueden llevarse de un lugar a otro, y a los incorporales referidos a los mismos, los signos distintivos, invenciones, derechos de autor, derechos de llave y similares, las naves y aeronaves, así como los documentos y títulos cuya transferencia implique la de cualquiera de los mencionados bienes.

Como podrá apreciarse, dado que las criptomonedas son bienes muebles intangibles que no son similares a los señalados en el párrafo anterior, si una persona jurídica domiciliada permuta Bitcoins de su propiedad no se configurará una venta gravada con el IGV.

\subsection{Implicancias tributarias de la compraventa de criptomonedas}

En este caso tenemos que el Bitcoin sería transferido por su poseedor a un tercero a cambio de un precio en dinero o moneda de curso legal.

(19) Véase los principales aspectos de la referida Resolución Vinculante española: Resolución Vinculante de Dirección General de Tributos, V3625-16 de 31 de Agosto de 2016, https://www.iberley.es/resoluciones/resolucion-vinculante-dgt-v3625-16-31-08-2016-1441124

(20) Si bien escaparía a los alcances del presente artículo, que es más bien de carácter introductorio sobre las criptomonedas, se podría evaluar si es posible considerar que nos encontramos ante una exportación de servicios en esta fase de minado de Bitcoins. 
Bitcoin: ¿Tenerla o ignorarla? Una aproximación hacia el tratamiento tributario peruano de la criptomoneda más famosa del mundo

Bitcoin: Have it or ignore it? An approach to Peruvian tax treatment of the most famous cryptocurrency in the world

Es importante destacar que, para efectos tributarios, ante la ausencia de una definición expresa de valor mobiliario, y en aplicación de la Norma III del Título Preliminar del Código Tributario, es posible recurrir a otras fuentes del Derecho (como la Ley de Títulos Valores). De este modo, enfatizamos que el Bitcoin no es un valor mobiliario, según explicamos en la sección 3.5 del presente artículo.

\subsubsection{Impuesto a la Renta (IR)}

La venta del Bitcoin generará renta gravada para su transferente persona jurídica domiciliada sobre la diferencia entre el valor de transferencia (precio de venta, que no podrá ser inferior al valor de mercado de la criptomoneda), menos el costo computable del Bitcoin (el que dependerá de si para su obtención se hizo uso de minado, de un intercambio o compra bursátil). En caso la transferencia sea realizada ante una sociedad vinculada, aplicarán las reglas de precios de transferencia. La tasa del IR aplicable a dicho transferente será del $29.5 \%$ sobre renta neta anual.

Cabe indicar que si las Bitcoins son transferidas por personas naturales domiciliadas (que no realizan actividad empresarial vinculada a las criptomonedas), dicha operación no estará gravada con el IR en Perú, dado que estas solo generan ganancias de capital gravadas en el caso de venta de inmuebles y de valores mobiliarios (que no es el caso de los Bitcoins).

En este punto es relevante indicar que no existiría un criterio de habitualidad objetivo (basado en un número de ventas en un periodo, por ejemplo) aplicable para el caso de la compra y venta de Bitcoins por personas naturales. No obstante, podría ser discutible que una persona natural que adquiere Bitcoins solo para su enajenación (especulación) pueda ser de plano descartada como alguien que realiza una actividad habitual; y por tanto, empresarial.

Por ello, debería analizarse la pertinencia de la inclusión de las criptomonedas dentro de la definición de valores mobiliarios que se encuentra en el literal a) del artículo 2 de la Ley del IR. De esta forma, las personas naturales que regularmente compran y venden bitcoins y demás criptomonedas estarían sujetas a tributación por las rentas de estas actividades como rentas del capital, mas no como rentas empresariales.

En el caso de transferentes no domiciliados (personas naturales o jurídicas) que no realizan actividad empresarial. En efecto, dichos sujetos solo pagarán IR por sus rentas de fuente peruana, y en la medida que las criptomonedas no son valores mobiliarios (no aplicamos las reglas del literal h) del artículo 9 de la LIR), y además dicho bien incorporal no sería utilizable económicamente en Perú, entonces la venta de dichos bienes muebles incorporables no estará gravada con el IR para los sujetos no domiciliados.

\subsubsection{IGV}

Tal como indicamos en el supuesto anterior, la venta de un Bitcoin no calificará como una operación gravada para el IGV al no formar parte de los bienes muebles cuya transferencia en propiedad está gravada.

\subsection{Experiencia tributaria comparada}

A continuación, recogeremos brevemente las consideraciones tributarias respecto a las criptomonedas en los tributos comparables al IR e IGV (Impuesto al Valor Agregado o IVA en otros países), aplicadas en jurisdicciones distintas a la peruana:

\subsubsection{Argentina ${ }^{(21)}$}

A partir del año 2018, se regula expresamente que se grava con el Impuesto a las Ganancias (símil al IR peruano), las rentas derivadas de las monedas digitales la tasa impositiva y la oportunidad del gravamen dependerá de si el depósito de las criptomonedas se encuentra en Argentina (billetera virtual) o en el exterior.

De otro lado, las operaciones con criptomonedas no son alcanzadas por el IVA, al ser un bien inmaterial que no califica como ninguna de las operaciones que sonalcanzadas por el IVA. Si bien las comisiones y cargos que se paguen por operaciones con criptomonedas sí se encuentran gravadas.

\subsubsection{México(22)}

México es el primer país que estrena una Ley para Regular las Instituciones de Tecnología

(21) Para estos efectos, revisar:Sitio web de AFIP, Impositiva Aduana Seguridad Social, https://www.afip.gob.ar/sitio/externos/default.asp (consultada el 10 de enero de 2019); http://impuestosblog.com.ar/afip-criptomonedas-que-impuestos-tributa-el-bitcoin/(consultada el 10 de enero de 2019); Guido Antolini, "Bitcoin y las monedas virtuales en el derecho argentino. ¿Qué son y cómo están reguladas?, Repositorio de la Universidad de San Andrés, http://repositorio.udesa.edu.ar/jspui/bitstream/10908/15619/1/\%5BP\%5D\%5BW\%5D\%20 T.\%20G.\%20Abo.\%20Antolini\%2C\%20Guido.pdf(consultada el 10 de enero de 2019); Martina Caunedo, "Una abogada experta en criptomonedas explica qué impuestos hay que pagar en la Argentina", sitio web de Cripto, https://www.infobae.com/cripto247/ altcoins/2018/07/20/una-abogada-experta-en-criptomonedas-explica-que-impuestos-hay-que-pagar-en-la-argentina/(consultada el 10 de enero de 2019); Martina Caunedo, "Según el borrador de la AFIP, las criptomonedas pagarán ganancias en Argentina", sitio web de Cripto,https://www.infobae.com/cripto247/altcoins/2018/08/14/segun-el-borrador-de-la-afip-las-criptomonedas-pagaranganancias-en-argentina/(consultada el 10 de enero de 2019).

(22) Revisar: Poblanerías.com, “Criptomonedas: ¿se pagará impuesto en México?”, sitio web de Poblanerías.com, https://www.poblanerias. 
Financiera ("Fintech"), publicada el 9 de marzo de 2018. Si bien la Ley es general, y no establece un tratamiento fiscal particular a las transacciones con criptomonedas, ciertas transacciones pueden derivar implicaciones fiscales, entre estas la permuta.

La figura aplicable al intercambio de bienes o servicios por un Bitcoin es el de la permuta la cual se encuentra regulada por el Título Tercero del Código Civil Federal. Dicho ordenamiento considera a la permuta como una compraventa.

Por lo tanto, una permuta de bienes o servicios por Bitcoins puede ser considerada para efectos fiscales como una enajenación de bienes, la cual puede conllevar un acto gravado con el IVA y el Impuesto sobre la renta en las dos partes involucradas en la permuta.

\subsubsection{Brasil}

A partir de marzo de 2014, Brasil incluye a las criptomonedas en la categoría de activos financieros aplicándoles un tipo de gravamen del $15 \%$ a las ganancias de capital resultantes, pero sólo exigiéndose a ventas superiores a un margen, quedándose exentas las transacciones de Bitcoins que involucren montos no significativos (Ramírez 2018, 21).

\subsubsection{España ${ }^{(23)}$}

El Instituto de Contabilidad y Auditoría de Cuentas considera que las monedas virtuales son inmovilizados intangibles en la medida en que no se destinen a la venta en el curso ordinario de las operaciones de su poseedor, en ese caso serán consideradas como existencias.

Al tratarse a las criptomonedas como activos, entonces los intercambios con monedas virtuales (o de Bitcoins con otros bienes distinto al dinero) califican como permutas de bienes.

En el caso del minado de Bitcoins, se considera que en la medida en que el "minero" está generando un ingreso mediante el uso de medios propios, este es considerado como un rendimiento de actividad económica, siendo deducibles los gastos relacionados a dicha actividad (amortización de los equipos informáticos, electricidad, etc.).
Finalmente, respecto al IVA la transferencia de Bitcoins se encuentra exenta ya que la normativa la de Comunidad Europea prescribe que la moneda virtual para dichos efectos (del IVA) es un medio de pago. De igual forma, a efectos de IVA, el minado se considera como operación sujeta, pero exenta de dicho tributo.

\subsubsection{Estados Unidos en América}

De acuerdo con la Administración Tributaria americana (el "IRS"), las monedas virtuales son consideradas como bienes para efectos de la tributación federal (IRS 2018). Consecuentemente, el intercambio de estos por otros bienes distintos al dinero genera una potencial renta gravada con el Impuesto sobre la Renta, como una permuta de bienes.

La definición de la transferencia como generadora de ganancia de capital de corto o largo plazo dependerá del tiempo de tenencia del Bitcoin por su transferente (superior a un año).

En el caso del minado de criptomonedas, se considera que dicha actividad causa renta gravada para el minero representada por el valor de mercado del Bitcoin el día en que es emitido como consecuencia del proceso de minado (Dordan y Charreau 2018, 3).

\subsubsection{Francia}

De acuerdo con una resolución del Consejo de Estado y la Jurisdicción Administrativa de abril de 2018, sobre el tratamiento fiscal de los Bitcoins, se parte de la afirmación que los Bitcoins son considerados como bienes muebles intangibles según el Código Civil francés. Según ello, la disposición de Bitcoins

com/2018/03/criptomonedas-se-pagara-impuesto-en-mexico/ (consultada el 12 de enero de 2019); Secretaría de Gobernación (SEGOB), Decreto por el que se expide la Ley para Regular las Instituciones de Tecnología Financiera y se reforman y adicionan diversas disposiciones de la Ley de Instituciones de Crédito, de la Ley del Mercado de Valores, de la Ley General de Organizaciones y Actividades Auxiliares del Crédito, de la Ley para la Transparencia y Ordenamiento de los Servicios Financieros, de la Ley para Regular las Sociedades de Información Crediticia, de la Ley de Protección y Defensa al Usuario de Servicios Financieros, de la Ley para Regular las Agrupaciones Financieras, de la Ley de la Comisión Nacional Bancaria y de Valores y, de la Ley Federal para la Prevención e Identificación de Operaciones con Recursos de Procedencialícita, Diario Oficial de la Federación, http://www.dof.gob. $\mathrm{mx} /$ nota_detalle.php?codigo=5515623\&fecha=09/03/2018(consultada el 12 de enero de 2019); Banco de México, "Reporte sobre el Sistema Financiero", http://www.banxico.org.mx/publicaciones-y-prensa/reportes-sobre-el-sistema-financiero/\%7B0B4861B7-28107FE9-88FE-2427C182512A\%7D.pdf(consultada el 12 de enero de 2019);Banco de México, "Comunicado de Prensa: Advertencias sobre el uso de activos virtuales como sucedáneos de los medios de pago en moneda de curso legal", http://www.banxico.org.mx/ publicaciones-y-prensa/miscelaneos/\%7B881612EF-DEC3-E03E-8C5A-C795CA66ACEA\%7D.pdf(consultada el 12 de enero de 2019).

(23) Respecto a España revisarhttps://reunir.unir.net/bitstream/handle/123456789/6521/ONRUBIA\%20DIAZ\%2c\%20JAIME pdf?sequence=1\&isAllowed=y; Angels Niubó, "Bitcoins: implicaciones fiscales", sitio web de Deloitte, https://www2.deloitte.com/es/ es/pages/legal/articles/bitcoins-implicaciones-fiscales.html; Fleur Dordan y Fiona Charreau, "L'imposition des Bitcoins, la France précise les modalités”, Revue OFIS, https://www.pantheonsorbonne.fr/fileadmin/diplome_M2OFIS/OFIS_2017-2018/article-bitcoinfinal.pdf 
Bitcoin: ¿Tenerla o ignorarla? Una aproximación hacia el tratamiento tributario peruano de la criptomoneda más famosa del mundo

Bitcoin: Have it or ignore it? An approach to Peruvian tax treatment of the most famous cryptocurrency in the world

puede generar ganancias de capital gravadas con el impuesto a la renta (l'impôt sur le revenu). Ahora bien, la adquisición y venta de Bitcoins ejercida de forma habitual y por cuenta propia calificará como beneficio industrial y comercial (Dordan y Charreau 2018, 6-7).

Tratándose de la actividad de minado de Bitcoins, el Consejo de Estado considera que esta no implica una adquisición propiamente dicha de Bitcoins; $y$, por tanto, no genera una compra y venta de las criptomonedas, las ganancias resultantes del minado son calificadas bajo la categoría de beneficios no comerciales $^{(24)}$

Finalmente, respecto al IVA la transferencia de Bitcoins se encuentra exenta ya que la normativa la de Comunidad Europea prescribe que la moneda virtual para dichos efectos (del IVA) es un medio de pago.

\subsubsection{Canadá(25)}

Según las directivas de la Agencia de impuestos canadiense (l'Agence du revenu du Canada), las criptomonedas son consideradas como cualquier otro bien para efectos impositivos (no se trata como dinero). De esta forma, las reglas de tributación que aplica a su intercambio por bienes o servicios son las del trueque o permuta.

Tratándose del minado de Bitcoins, se precisa que cuando esta actividad se desarrolle de manera comercial y con espíritu empresarial, el valor de las criptomonedas recibidas será incluido como rentas gravadas para fines del impuesto a la renta. Paralelamente, los gastos del minero en electricidad y equipos informáticos podrán ser aplicados contra las referidas rentas.

\section{Conclusión}

El arribo del fenómeno de las criptomonedas es una realidad en el Perú. Los siguientes hechos dan cuenta de ello: el crecimiento acelerado del número de establecimientos que aceptan dichas monedas virtuales como medios de intercambio de bienes y servicios; el pago de remuneraciones y compensaciones en criptomonedas por diversos empleadores, sobre todo por las Fintech; la aparición de Exchange locales y cajeros automáticos de Bitcoins.

Sin embargo, y como no es novedad, la regulación legal expresa de este tipo de innovaciones tecnológicas que tienen un doble fin: medio de pago y/o instrumento de inversión (especulativo o no), es a la fecha nula en nuestro país.
El interés internacional hacia la regulación de las criptomonedas está enfocado principalmente a prevenir delitos asociados a estas monedas virtuales, como son los de lavado de activos, financiamiento al terrorismo, y la evasión fiscal.

Respecto al tratamiento tributario aplicado a los Bitcoins en la legislación comparada, esta tiende mayoritariamente a clasificarlos como bienes muebles, cuya negociación genera los efectos de una permuta; y su obtención mediante el minado, es considerado como un servicio remunerado en especie (los Bitcoins emitidos).

En el Perú,la carencia de un tratamiento legal expreso de los Bitcoins no impide que podamos utilizar las definiciones legales existentes para perfilar un tratamiento tributario a las operaciones relacionadas con las criptomonedas y a sus protagonistas: minado o producción, intercambio por bienes o servicios, o su enajenación a cambio de monedas de curso legal.

A pesar de ello, una regulación tributaria(o legal) específica de las criptomonedas permitiría el escenario siempre más saludable de la certidumbre, dentro del cual se puede incentivar el crecimiento del uso de las criptomonedas para ámbitos no solo de inversión sino también de la vida cotidiana.

\section{Bibliografía}

Dordan, Fleur y Charreau, Fiona. L'imposition des Bitcoins, la France précise les modalités. Revue OFIS. https://www.pantheonsorbonne. fr/fileadmin/diplome_M2OFIS/OFIS_20172018/article-bitcoin-final.pdf

GAFI. Véase Grupo de Acción Financiera. Informe del GAFI. Monedas Virtuales: Definiciones claves y riesgos potenciales de LA/FT. https://www.uaf.cl/asuntos/descargar. aspx?arid=961 (consultada el 15 de febrero de 2019).

(24) Para revisar la referida resolución del Consejo de Estado Francés: Sitio web de Conseil d'État, http://www.conseil-etat.fr/DecisionsAvis-Publications/Decisions/Selection-des-decisions-faisant-I-objet-d-une-communication-particuliere/Conseil-d-Etat-26-avril-2018M.-G-et-autres

(25) BDO Canadá, "La position de l'ARC sur les cryptomonnaies : incidence sur l'impôt sur le revenu“, sitio web de BDO Canadá, https:// www.bdo.ca/fr-ca/insights/tax/tax-articles/cra-cryptocurrency-implications/ 
Gil Soriano, Alberto. Monedas virtuales: Aproximación jurídico - tributaria y control tributario. Actualidad Jurídica Uría Menéndez. https://www.uria.com/documentos/ publicaciones/5803/documento/foro_esp_02. pdf?id=7879 (consultada el 10 de enero de 2019).

Gutiérrez, Omar y Moreno, Abraham. 2018. El bitcoin: consideraciones financieras y legales sobre su naturaleza y propuesta de enfoque para su regulación. Lima: ESAN ediciones.

IRS. Véase Internal Revenue Service. IRS reminds taxpayers to report virtual currency transactions. Sitio web de IRS. https://www. irs.gov/newsroom/irs-reminds-taxpayers-toreport-virtual-currency-transactions.
Montoya Manfredi, Ulises, Montoya Alberti, Ulises y Montoya Alberti, Hernando. 2004. Derecho comercial. Tomo II. Lima: Editora Jurídica Grijley.

Szczepanski, Marcin. Bitcoin: market, economics and regulations. European Parliamentary Research. http://www.europarl. europa.eu/RegData/bibliotheque/briefing/2014/140793/ LDM BRI(2014)140793_REV1_EN.pdf (consultada el 20 de febrero de 2019).

RamírezGracia, Víctor. Hacia un tratamiento uniforme del Bitcoin desde una perspectiva fiscal global. Tesis para el Máster en Fiscalidad Internacional. Universidad Internacional de La Rioja. Sevilla, 2018. Página 8.

Sotiropoulou, Anastasia y Guégan, Dominique. 2017. Bitcoin and the challenges for financial regulation. Capital Markets Law Journal, Volume 12, Number 4 (octubre): 466-479. https://doi. org/10.1093/cmlj/kmx037. 
Bitcoin: ¿Tenerla o ignorarla? Una aproximación hacia el tratamiento tributario peruano de la criptomoneda más famosa del mundo

\section{Anexo: Infografía del tratamiento tributario de los Bitcoins en el Perú Criptomonedas/Bitcoins}

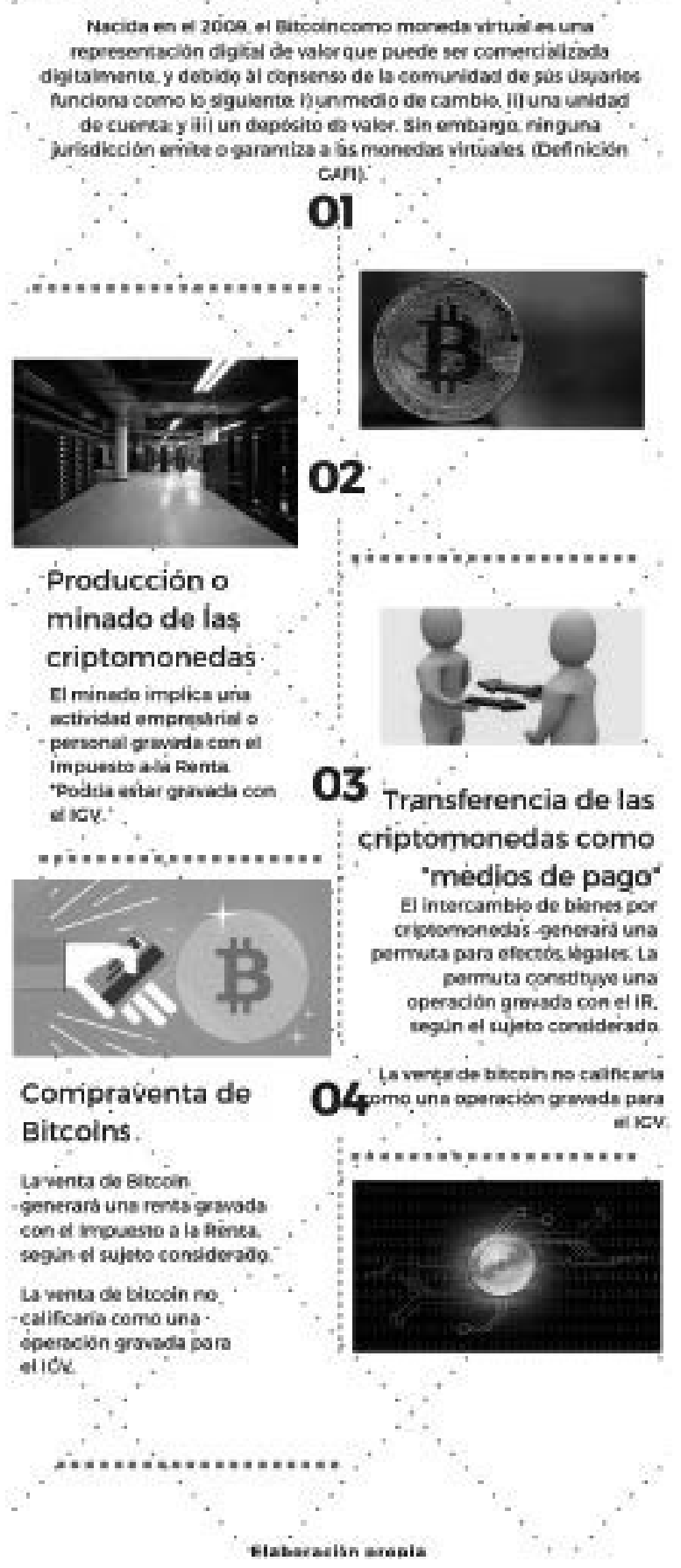

